\title{
Study on the Characteristic of Eye During
}

\section{Traffic Operation}

\section{Xu Hui-zhi ${ }^{1, a}$, Wang Yu-ping ${ }^{2, b}$, Wu Chong ${ }^{1}$, Cheng Guo-zhu ${ }^{3}$, Pei Yu-long ${ }^{3}$ \\ ${ }^{1}$ Post-doctoral Station of Management science and engineering, Harbin Institute of Technology,}

Harbin 150001, China, ${ }^{2}$ Harbin Metro Group Corporation Ltd, Harbin 150000, China, ${ }^{3}$ School of

Transportation Science and Engineering, Harbin Institute of Technology, Harbin 150090, China

a353812396@qq.com, b rock510@163.com

Key words: Traffic Operation; Driver; Characteristic of Eye; Experiment

\begin{abstract}
To design vehicle auxiliary equipment, it need master the characteristic of eyes. The paper adopted iView X HED as equipment to get the characteristic of eye during traffic operation, results could be treat as experiment research. The paper collected text data of eye, and adopted location, during, blink time, blink frequency, and transient of eye focus as parameters to study the characteristic of eye, and given a measurable value to them. It was a foundation to further research of driver's characteristic. The result showed that: the location mainly focus in ahead, the average value of time and blink was $0.3098 \mathrm{~s}$ and $0.231 \mathrm{~s}$. Frequency of blink was respond to traffic condition, the mainly direction of transient was left and right.
\end{abstract}

\section{Introduction}

Traffic flow could produce traffic conflict. Mainly reason was the drivers got inadequate information about traffic environment. ITS proposed a concept of assistance system to driver, but need to know the characteristic of drivers, especially to the characteristic of driver's eye ${ }^{[1,2]}$. Then, it could design an equipment of information collection and dangers warning ${ }^{[3,4]}$. Such, the paper design a test to study the characteristic of eye, via by collected data of location, during, blink time, blink frequency, transient of eye focus, to quantitative study to characteristic of eye in traffic ${ }^{[5 ; 6]}$.

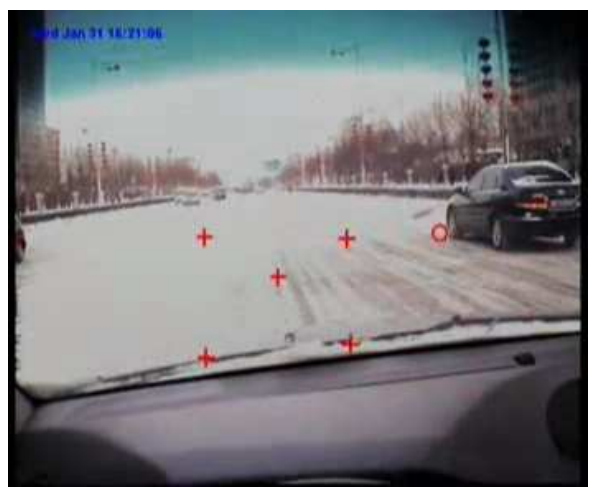

Fig1. Investigate scenes1



Fig2. Investigate scenes2 
The equipment of information collection was iiView X HED. Principle of work was got the eye' s image by infrared camera, and to track the trajectory of eye' $\mathrm{s}$ focus. Real-time computing the time, distance and speed of eye in Horizontal and vertical direction, and computing the location and diameter of pupils. Sampling rate was $50 / 60 \mathrm{~Hz}$, Tracking resolution was $0.1^{\circ}$, Monitor accuracy of recording was $0.5 \sim 1^{\circ}$, and the weight of helmet was $450 \mathrm{~g}$. The connection had two method: Wired and wireless (wireless connection was Bluetooth, the max control distance was $300 \mathrm{~m}$ ). Fig1 and fig2 was the special scene in traffic survey. Cross was the calibrated point and the circle was the location of driver' $s$ view-point in traffic survey scene ${ }^{[7,8]}$.

\section{Location and During of View-point}

During the driving, drivers transit their view-point corresponding to the change of traffic environment. In the proceeding, the view-point would remain in a stable point, and collected the information of traffic environment and to make a judgment, then, to decide the operating characteristic in next step. It could get from the traffic survey; the parameter of view-point during reflected the capacity of driver to concept and deal information. The average during of view-point was 700.4s. The mount of simple was 1233 .

Location of View-point. Distribute of view-point location were shown in fig3 when driving in city road. So, it could get the conclusion: the distributed of view-point was mainly in the center of the field view-area.

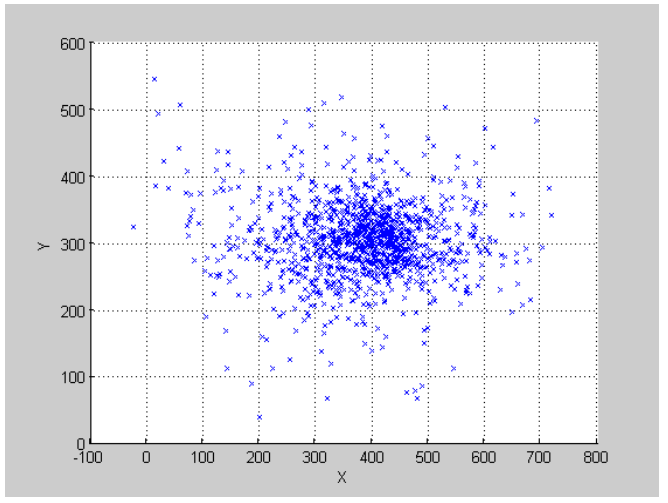

Fig3. Distribution of the view-focus location

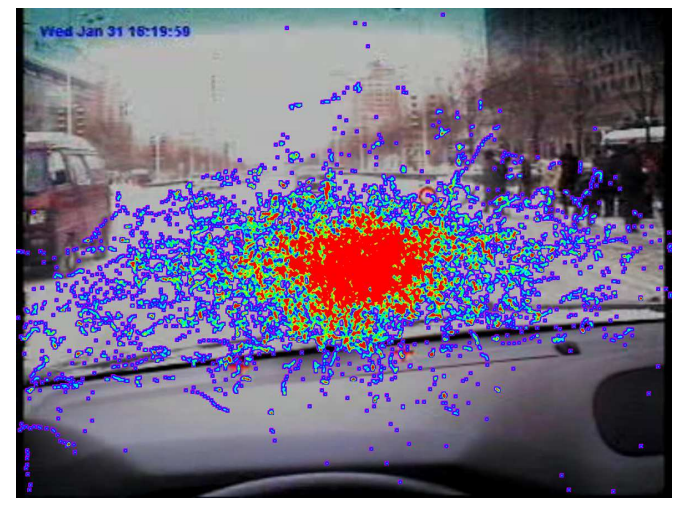

Fig4. Heat of the view-focus location

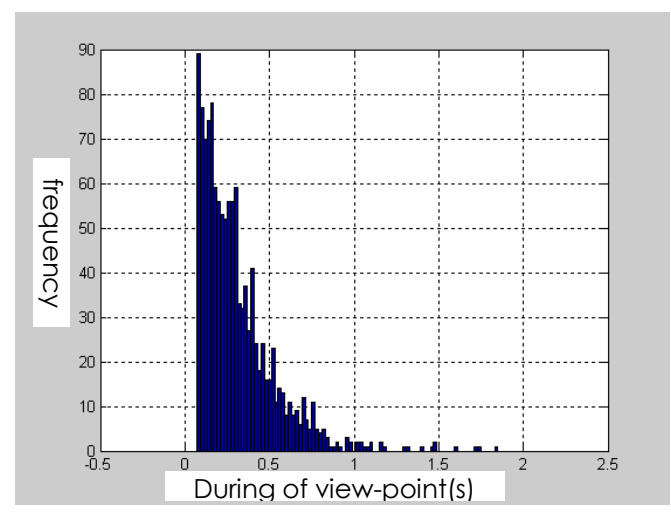

Fig5. Frequency of the viewpoint residence time 
During of View-point. It divides the collection data of view-point during into $0.02 \mathrm{~s}$ groups, then to draw the frequency chat shown in fig5. The max value was $1.840 \mathrm{~s}$, the min value was $0.078 \mathrm{~s}$, and the average value was $0.3098 \mathrm{~s}$. The frequency was 89 in the interval $(0.07 \mathrm{~s}, 0.09 \mathrm{~s}], 77$ in the interval $(0.09 \mathrm{~s}, 0.11 \mathrm{~s}]$, and 70 in the interval $(0.11 \mathrm{~s}, 0.13 \mathrm{~s}]$. Overall, the frequency of view-point during reduced corresponds to the increased of operation time.

\section{Blink time and Blink Frequency}

Blink time and blink frequency were indicators which reflect the physiological characteristic of drivers. It could get the capacity of drivers to receiving and processing information. The length of survey dada was 700.4s, and got 1413 simples of blink.

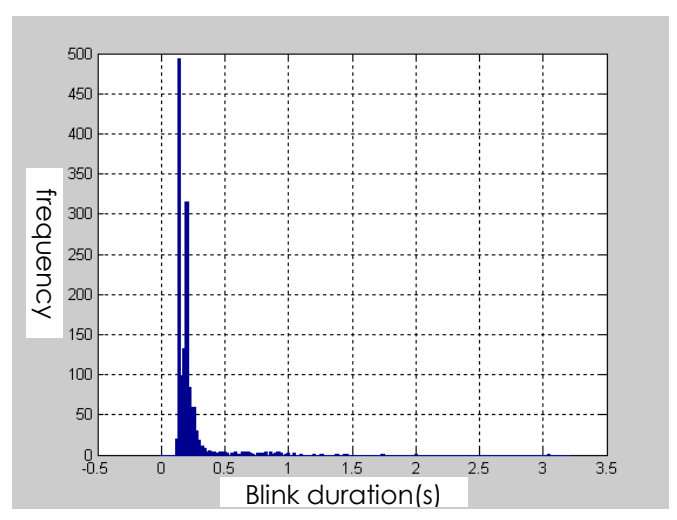

Fig6. Distribution frequency of blink duration

Blink Time. Blink time of drivers distribute in the interval [0.02s, 3.04s], the average value was $0.231 \mathrm{~s}$. It divide the blink time in several groups, the interval was $0.02 \mathrm{~s}$, draw the frequency chat shown as fig5.

The frequency was 493 in the interval $(0.13 \mathrm{~s}, 0.15 \mathrm{~s}]$, it was the first peak about the blink. The frequency was 315 in the interval $(0.19 \mathrm{~s}, 0.21 \mathrm{~s}]$, it was the second peak about the blink.

It could get the result that the frequency nearly equivalent in the interval $[0.35 \mathrm{~s}, 1.05 \mathrm{~s}]$, the frequency value in the interval $[1,4]$, the value was 0 or 1 in the interval $(1.05 \mathrm{~s}, \infty)$. Blink time mainly concentrated in the interval $[0.11 \mathrm{~s}, 0.33 \mathrm{~s}]$.

Blink Frequency. The blink frequency was real time change during operation. Operation time and environment were main reason to change the blink frequency. Thus, it could get the fatigue characteristic of drivers though the parameter of blink frequency.

It got the statistics table via by survey data, as shown in fig 1, and take parameters such as average value, max value, min value, Mean square error. Average value reflected the number of blink, max value reflected the strength of blink, and Mean square error reflected the dispersion of blink. Min blink frequency was 0 times/10s, average was 2.213 times/10s.

Tab.1 Descriptive statistical indicators of blink data (10s)

\begin{tabular}{c|c}
\hline \hline index & value \\
\hline average value & 2.165 \\
\hline max value & 11.000 \\
\hline min value & 0.000 \\
\hline Mean square error & 2.213 \\
\hline \hline
\end{tabular}


It could get the result though analysis the statistical data: the driver need often blink to ease the fatigue witch cause by continued driving. On the other side, it also proves the correct that adopted blink frequency as the evaluated parameter to visual adaptability.

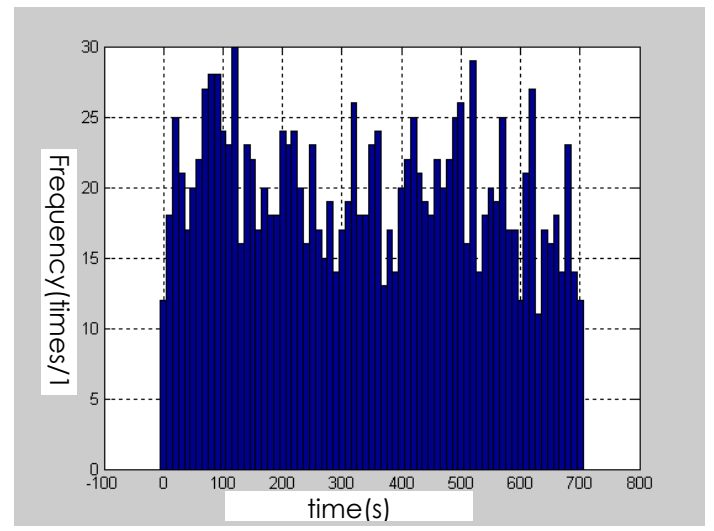

Fig7. Blink frequency

\section{Transient of Eye Focus}

The survey got 374 group data about transient of eye focus. It could draw a transient chat by the survey data in view field, as shown in fig9. Arrow direction reflected the direction of transient, Arrow size reflected the speed of transient. The transient of eye focus mainly occur in the center of view, but the transient speed was slowly relative to around field. The traffic environment and object continued change during driving, the drivers need to change location of view-point to collection the information. So, to study the regular pattern of eye focus transient could reflect the characteristic of driver correspond to the traffic change and urgent condition. In order to analysis the transient of eye focus, Enlarge the fig 9, and shown in fig10, fig11, fig12, fig13, fig14.

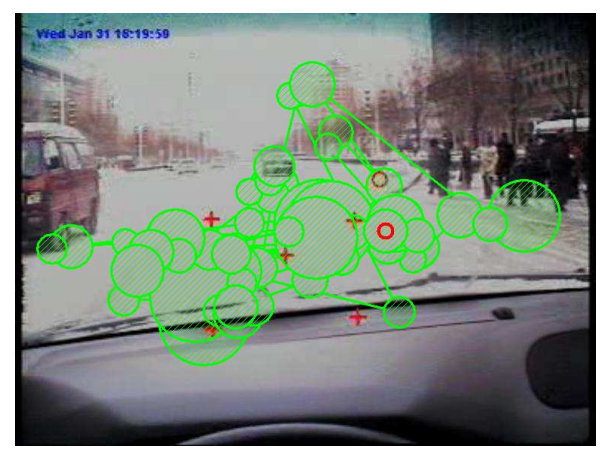

Fig8. Viewpoint stay and transfer

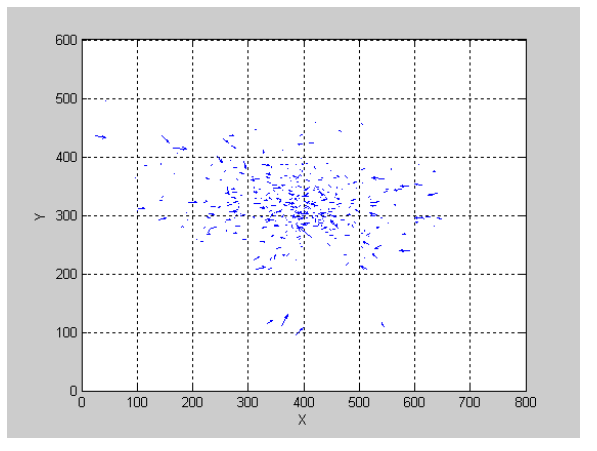

Fig9. Viewpoint quickly transfers

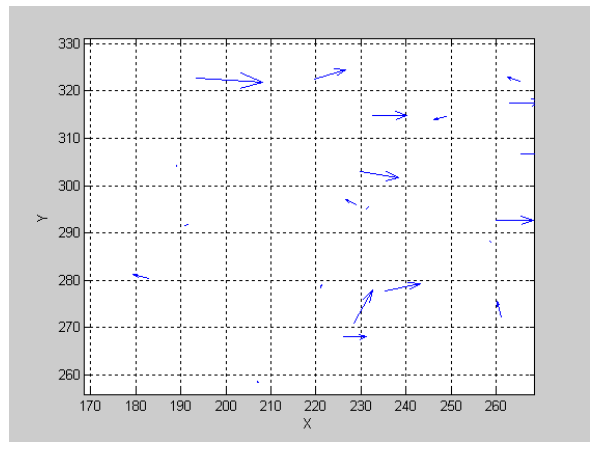

Fig10. Partial view of the left 


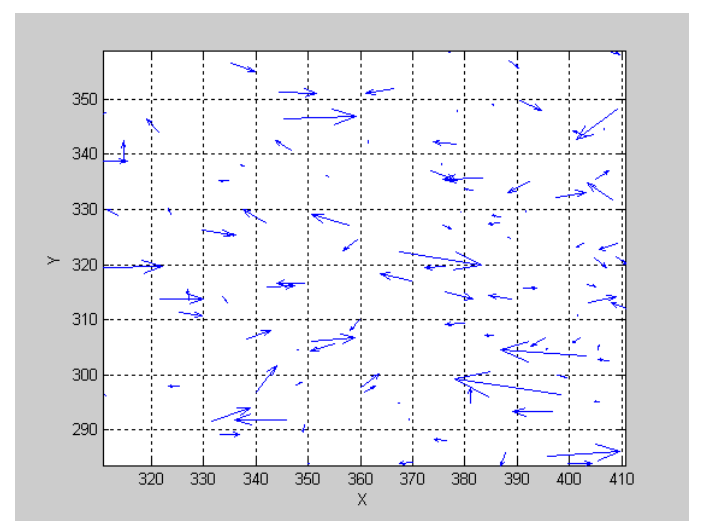

Fig11. Partial view of the center

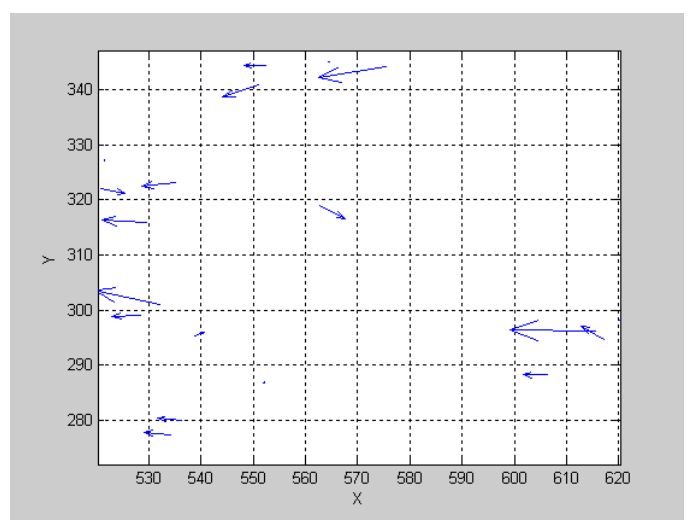

Fig12. Partial view of the right

It could know from fig10, the main direction of eye focus transient was right in left view field. In order to get the information in center and right area when eye focus in left, the speed of transient became fast, and more left, more fast.

It could know from fig11, the main direction of eye focus transient was left and right in center view field. Direction of up and down seldom appears. It transit became more frequency in the center view field.

It could know from fig12, the main direction of eye focus transient was left in right view field. The characteristic of eye focus transient was opposite to the left view field.

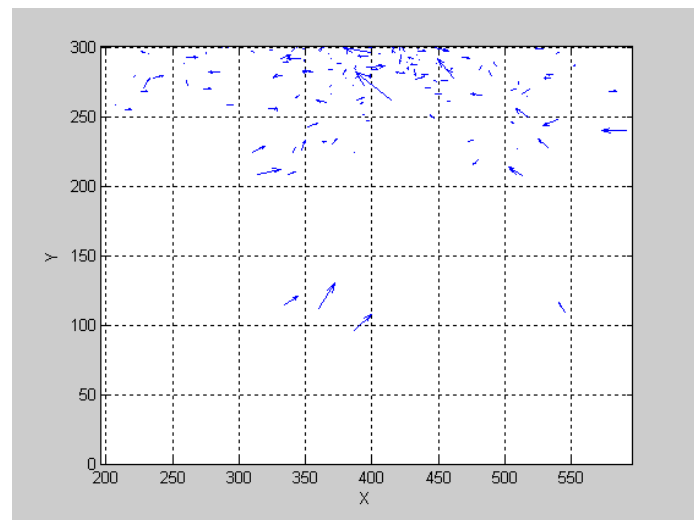

Fig13. Partial view of the lower

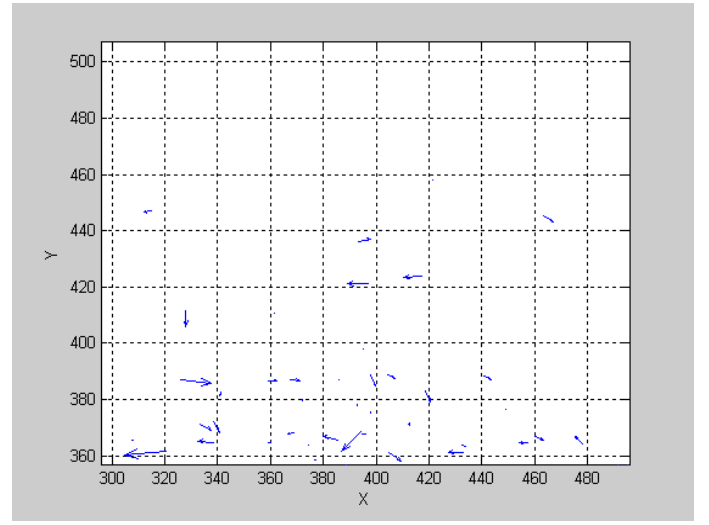

Fig14. Partial view on the side

It could know from fig13, the main direction of eye focus transient was down, left, right in up view field. The drivers though up view field to get the information in distant, the survey was city road, the average velocity was $20 \mathrm{~km} / \mathrm{h}$, the eye focus of driver was in $30 \sim 40 \mathrm{~m}$ ahead. The change in distant could not cause the rapid reflect, so, the characteristic of eye focus transient was less. In order to get the information in center and right area when eye focus in left, the speed of transient became fast, and more left, more fast.

It could know from fig14, the main direction of eye focus transient was up, left, right in down view field. The drivers though up view field to get the information in nearby, the driver was less attention to the information. The main direction was up, it reflect the characteristic that the driver transient his eye focus into center field when got the traffic information.

So, it could get the conclusions: the direction of eye focus transient was left and right. The more away to center field, the transient became more fast. 


\section{Conclusion}

It adopted the characteristic of eyes as study object, Quantitative analysis the parameters of location, during, blink time, blink frequency, transient of eye focus. The result shown that: the location of eye focus mainly in ahead, the average value of time and blink was $0.3098 \mathrm{~s}$ and $0.231 \mathrm{~s}$, frequency of blink was respond to traffic condition, the mainly direction of transient was left and right.

\section{Acknowledgements}

This work was financially supported by National Natural Science Foundation of China(51108137) and China Postdoctoral Science Foundation(2012M511499). Thanks for the sponsoring of the Fundamental Re-search Funds for the Central Universities (Grant No. HIT. NSRIF. 201150), and China Postdoctoral Science Foundation(20110491090).

\section{References:}

[1] M. J. Henning. Preparation for lane change manoeuvres: Behavioural indicators and underlying cognitive processes[D]. Ph.D. dissertation Fakultät Human und Sozialwissenschaften, TU Chemnitz, 2009.

[2] Dario D., Salvucci, Andrew Liu. The time course of lane change: Driver control and eye-movement behavior[J]. Transportation Research Part F 5, 2002, 5(2): 123-133.

[3] Hiroshi Ohno. Analysis and modeling of human driving behaviors using adaptive cruise control[C]. Applied Soft Computing, 2001, 1(3): 237-243.

[4] Yasushi Nishida. Driving characteristics of the elderly: risk compensation of the elderly driving from the viewpoint of reaction behavior[J]. JSAE Review, 1999, 20(3): 375-380.

[5] Ioannis Golias, Mstthew G., Karlaftis. An international comparative study of self-report driver behavior[J]. Transportation Research Part F, 2002, 4(4): 243-246.

[6] Nakaho Numata, Hiroki Kitajima, Yoshihiro Goi, Keiichi Yamamoto. Analysis of driver's behavior before and after crashes in simulated expressway driving to predict sleeping levels for doze alarm activation[J]. JSAE Review, 1998, 19: 269-275.

[7] Jerry L. Deffenbacher, David M. Deffenbacher, Rebekah S., Lynch. Anger, aggression, and risky behavior: a comparison of high and low anger drivers[J]. Behavior Reach and Therapy, 2003, 41(6): 701-718.

[8] Mohamed A., Abdel-Aty, A., Essam Radwan. Model traffic accident occurrence and involvement[J]. Accident Analysis and Prevention, 2000, 32(5): 633-642. 